\title{
Il sacrificio di Orfeo nell'Orfeo del Poliziano
}

La Fabula di Orfeo viene generalmente ricordata per il suo valore storico nel quadro della tradizione drammatica italiana, quale prima rappresentazione in volgare di un soggetto profano, in quanto sostituisce un'azione mitologica ad un'azione sacra, e quale primo esempio di dramma pastorale del Rinascimento. Le si riconosce anche valore letterario e filologico, presentato dalla forma ibrida della composizione che, sotto l'amalgame di elementi diversi, realizza a pieno l'ideale artistico della docta varietas, la nota tecnica enunciata dal Poliziano nella Prolusione a Stazio e Quintiliano, nei Nutricia, nel Panepistemon, e nella prefazione ai Miscellaneorum Centuria Prima. Effettuandosi mediante un processo di imitatio e di contaminatio, la docta varietas, in effetti, ricrea l'argomento ed i personaggi del mondo vagheggiato nelle Bucoliche e nelle Georgiche e raggiunge, nell'Orfeo, attraverso il filtro della cultura classica, la piú alta espressione, la trasfigurazione, e la sintesi di quanto, nel Quattrocento italiano, ci fosse di piú dotto, raffinato, e letterario. ${ }^{1}$ L'imitazione è soltanto apparente; in realtà, tutte le fonti a cui ha attinto vengono investite e quindi trasformate dal poeta. La molteplicità di reminiscenze ovidiane e virgiliane - vergiliane, insisterebbe il Poliziano ${ }^{2}$ - le movenze catulliane o dantesche, i residui stilnovistici, guittoniani, o petrarcheschi, gli echi popolareschi e le suggestioni derivate dalle momarie, ${ }^{3}$ sono assimilati genialmente e risolti in una costruzione levigata ed armonica e in un effetto di "varietà e di incisiva drammaticità, ma . . . vistosamente contenute e frenate dalla scoperta e [si direbbe] ostentata stilizzazione letteraria. ${ }^{\prime 4}$

A parte il fatto di riconoscere il valore storico e letterario della Fabula, la critica, come si è lamentato il Russo, tende un po' troppo facilmente ad accentuare la qualità "aerea, musicale ... iperuranica" della poesia del Poliziano, "quasi volesse trasumanar[la] e trasportarla in un cielo irreale. ${ }^{5} \mathrm{Il}$ fatto che, in apparenza, 
il senso tragico sia eluso con l'abbandono al tono ora elegiaco, ora lirico, ora oratorio, ora orgiastico ha indotto a ridurre tutta l'opera ad un senso o fiabesco, o idillico, o musicale o spettacolare. ${ }^{6}$ Solo recentemente, il Bigi ha rilevato ed illustrato finemente la "materia etica" ed umana dell'Orfeo, che si esprimerebbe attraverso il tema dell' "irrazionalità dell'amore," di cui mette in evidenza, appunto, la "violenza irrazionale e distruggitrice."7

Questi giudizi, pur mettendo in risalto aspetti rilevanti dell'opera, tendono, se non a ridurre, almeno ad attenuare la sua originalità, trascurando altri aspetti importanti della Fabula, primo fra tutti il significato ideologico del contenuto. Il Garin, che pare avere individuato un certo legame ideologico tra l'Orfeo ed alcuni versi dei Nutricia, ${ }^{8}$ e che ravvisa nel mito di Orfeo "il simbolo della parola educatrice che costituisce l'uomo a se stesso cementando le società, e poi facendolo signore delle cose, ${ }^{\prime 9}$ non allude pertanto che all'episodio del dominio esercitato dal canto di Orfeo sulle divinità infernali, senza fare riferimento alcuno al resto dell'opera.

La Fabula di Orfeo, araldo dell'opera e del teatro italiano, apice e conclusione della piú splendida attività poetica del Poliziano, è anche la sua piú alta esaltazione del ruolo della poesia, simboleggiato dal potere della parola del poeta e dal sacrificio di Orfeo, che effettua l'ideale della ricreazione secondo l'humanitas.

Innanzitutto, bisogna chiedersi perché il Poliziano abbia scelto il mito di Orfeo per soggetto della Fabula. Esso rientrava nel suo consueto mondo poetico, sia ispirato alla narrazione virgiliana ed ovidiana, sia per lo sfondo pastorale della vicenda, già ritratto nelle Stanze. Aveva rievocato Orfeo nell'Elegia al Fonzio, quale "Apollinei modulator carminis," 10 e lo rievocherà ancora nella Manto e nei Nutricia, dove traccia la storia ideale dell'educazione sotto l'egida dei poeti, creatori di una nuova, profonda, ed umana moralità e dignità. ${ }^{11}$ Non è sorprendente inoltre che abbia scelto il mito di Orfeo, considerando la sua diffusione tra gli umanisti neoplatonici del Quattrocento. Basti ricordare il Ficino, per cui Orfeo rappresenta colui che organizza la creazione mediante una doppia intuizione, la filosofica e la poetica. L'elogio dell'Orfeo teologo, in Ficino, quale vate dell'antichità, fondatore dei misteri, ${ }^{12}$ non esclude né l'interpretazione neoplatonica - secondo la quale Orfeo rappresenta il simbolo del dominio dell'anima sul mondo, del poeta che crea l'armonia universale mediante il potere della parola e della musica ${ }^{13}$ — né l'interpretazione cristiana - nell'immaginare Dio come un pastore orfico che veglia sul mondo. ${ }^{14} \mathrm{Ma}$ anche, molto probabilmente, il Poliziano sentiva sue, piú degli 
altri membri dell'Accademia ficiniana, le sventure del poeta tracio: sia il ruolo della poesia che il rapporto del poeta stesso con la morte lo toccavano direttamente. ${ }^{15}$ Cosí il mito di Orfeo diventa il veicolo di espressione della meditazione del poeta: la "favola" e lo spettacolo non ne sono che la cornice, sotto la cui geniale stilizzazione artistica si vela il significato profondo e allusivo, proprio come nelle teologie antiche, che si esprimevano in favole poetiche affinché i misteri religiosi non potessero essere rivelati al profano.

Nonostante il Poliziano avesse voluto che la Fabula rimanesse sconosciuta, ${ }^{16}$ è proprio ad essa che affidava il risultato di una lunghissima ed intensa preparazione spirituale: ridimensiona l'antico mito in un quadro umano e, riscoprendone ed interpretandone i motivi, lo fa assurgere a simbolo universale della condizione del poeta, ritratta nella sua piú intima e commovente tragedia.

La trama della Fabula, dalle linee stilizzate, e a tutti ben nota, è la seguente: Orfeo perde, ricupera, e perde di nuovo e definitivamente la sua amata Euridice; onde il suo odio per le donne e lo strazio delle Baccanti offese, che lacerano il suo corpo e, in un'orgiastica danza, innalzano un inno a Bacco, con cui si chiude la favola. Benché la vicenda suggerisca che si tratti di un dramma d'amore, un'attenta lettura dei versi ci convince che l'amore, elemento essenziale che sia, viene assorbito dalla piú alta concezione del Poliziano e trattato in funzione del suo intento principale, che è la meditazione sul rapporto tra il poeta, la poesia e la morte, rapporto che culmina e si risolve nel sacrificio di Orfeo.

Nella Fabula, il ruolo della poesia trascende l'ispirazione, espressa nelle Stanze, a conferire immortalità a "i gran nomi e i fatti egregi e soli" (I, 1, 7) e si esplica nella manifestazione e nella totale affermazione del suo carattere trionfale, personificate dal personaggio del poeta tracio.

Sin dalla sua prima apparizione, Orfeo è inquadrato nella cornice maestosa e trionfale della poesia, ed accompagnato dagli attributi apollinei della 'lira' e del 'canto,' ossia della poesia: appare "cantando sopra il monte in su la lira" (dopo il v. 137), dove la sua posizione privilegiata, da poeta, è accentuata, sul piano artistico, dalla posizione scenografica, sulla sommità del monte, che indica la sua superiorità sul resto dell'umanità. La saffica che recita, in latino, e in onore di Francesco Gonzaga, si apre con l'esortazione alla lira ad intonare una nuova poesia ("novum . . carmen"):

O meos longum modulata lusus

quos amor primam docuit iuventam, 
flecte nunc mecum numeros novumque

dic, lyra, carmen. (Vv. 138-41) ${ }^{17}$

L'ode latina, alla cui presenza si attribuisce generalmente solo senso encomiastico e cortigiano, ${ }^{18}$ costituisce, invece, un momento essenziale dell'azione, incarnando, nella dotta poesia latina, l'alta espressione di un ideale di cultura e di civilizzazione e, nel Signore di Mantova, generoso protettore dei poeti (possiede "doctas . . . aures . . . qui colit . . citharamque princeps," vv. 144-45, 147) e lui stesso poeta (congiunge le virtú di Mecenate e di Virgilio: "Mecoenas tibi nunc Maroque / contigit uni," vv. 180-81), la "rediciviva virtus" (v. 171), la virtú che si rinnova, di cui è degno erede ed emulo. Se Orfeo, fondatore della poesia e della civiltà, che vince la ferinità istintiva e primitiva, è il mito della humanitas, sia la poesia latina che il Gonzaga ne sono entrambi strumenti in quanto operano in funzione di essa.

Alla notizia della morte di Euridice, la prima reazione di Orfeo è quella di rivolgersi alla lira per intessere un nuovo canto:

Dunque, piangiamo, o sconsolata lira, ché piú non si convien l'usato canto

Forse che svolgerèn la dura sorte, co' lacrimosi versi, o dolce cetra. (Vv. 198-99; 208-09)

È con l'eloquenza della sua parola e la musica della sua poesia che riesce poi a ristabilire l'ordine nell'universo, nel dominare gli esseri infernali, di cui o ne arresta le attività abituali o ne provoca il movimento contrario, svolgendo, con il suo miracolo, ciò che il Russo ha definito un "mimo . . . drammatico"19 e che si fissa nelle immagini piú determinate dalle espressioni provocate dalla meraviglia di Plutone:

Chi è costui che con sí dolce nota muove l'abisso e con l'ornata cetra? Io veggo fissa d'Ission la rota, Sisifo assiso sovra la sua petra e le Belide star coll'urna vota, né piú l'acqua di Tantalo s'arretra, e veggo Cerber con tre bocche intento e le Furie acquetare al suo lamento. (Vv. 230-37) 
È dal suo 'canto' che Proserpina è persuasa: "pel canto, per l'amor, pe' giusti prieghi" (v. 293), dove, nell'ordine del discorso, la poesia (il 'canto') ha precedenza anche sull'amore stesso. Ed è al 'dolce plettro' che cede Plutone, riprendendo il filo di ragionamento della consorte: " $\mathrm{I}$ ' son contento che a sí dolce plettro / s'inchini la potenzia del mio scettro" (vv. 300-01).

Redenta Euridice, recita, di ritorno dall'inferno, versi latini che celebrano il trionfo della poesia sulla morte, canto della humanitas trionfante, esplicitamente reso dall'invocazione immediata al vittorioso alloro, dalla tripla ripetizione dell'espressione del trionfo in "triumphales," "triumpho" e "triumphe," della vittoria in "vicimus" e "victoria," della corona poetica in "Ite triumphales circum mea tempora lauri!," e del poeta stesso in "mea," "mihi," "mea" (vv. 302-05). Il canto è, in modo molto ovvio, la proclamazione della gloriosa vittoria della poesia sulla morte e non la celebrazione della riconquista della donna amata: Euridice sottratta al mondo dei morti non è altro che un pretesto, l'episodio che suggerisce solo il motivo iniziale. L'amore, come anche la bellezza, non sono esaltati come un qualcosa di assoluto e come fine a se stessi, ma visti con dimensioni umane, ritratti in una visione elegiaca e melanconica, e stagliati sullo sfondo della morte e del finito. Nel tentativo di Orfeo di volere rimirare ancora una volta il volto della sua amata, strappatagli prematuramente dalla morte "or la tenera vite e l'uva acerba / tagliata avete colla falce dura," vv. 272-73 - di sottrarre per un attimo la bellezza all'eterno fluire del tempo (il gran tema delle arti figurative del Quattrocento fiorentino) e nel tentativo di Aristeo di arrestare la fuggente "donzella" (v. 125), si dischiude pienamente il concetto per cui la bellezza interessa nella sua presenza immanente e non come mezzo per accedere alla piú intensa bellezza dello spirito, concetto che ricorre frequentemente nella letteratura del Rinascimento, nonostante gli insegnamenti delle teorie platoniche del tempo. Ugualmente, una melanconica consapevolezza che anche la bellezza di Euridice sarà sottoposta alla distruzione inesorabile del tempo sottolinea il noto motivo del carpe diem, nell'invito di Aristeo alla ninfa a godere della bellezza finché essa duri, espresso nei versi che accompagnano la "canzona pastorale" ("Digli, zampogna mia, come via fugge / cogli anni insieme suo belleza snella, / e digli come el tempo ne distrugge ..." vv. 70-75).

La concretizzazione della bellezza e dell'amore e la loro durata nel tempo fisico sono controllate da un principio posto al di fuori di esse, una forza esterna, sovrumana, che ne determina irrevoca- 
bilmente la fine; come ha scritto il Poliziano stesso: "Questa nostra vita non altrimenti sdrucciola che l'acqua d'un fiume; e le cose umane si dimenono un pezo, e finalmente rovinono e nessuno è che possi fuggire questa necessità che sopra il capo ci pende." 20 Ma la loro concretizzazione e la loro durata diventano eterne nel mondo atemporale e privilegiato dell'arte, controllato unicamente dalla parola del poeta. Euridice, simbolo dell'eterna primavera, immaginato e perseguito dall'uomo, esiste solo fino a quando c'è il poeta a darle vita; la sua esistenza è condizionata da quella del poeta che solo può infonderle vita e renderla eterna. La vera primavera è la poesia, il soggetto che essa ritrae fragile e caduco. Solo nella creazione artistica si esaurisce il supremo tentativo di liberazione dalla morte dal tempo e dalla fortuna, e l'amore e la bellezza passano dal mito alla realizzazione della vera eterna primavera.

Dopo la seconda morte di Euridice, la tragedia di Orfeo non è quella di Orfeo-uomo che ha perso la donna amata per sempre; la sua tragedia è quella di Orfeo-poeta, tragedia che sopraggiunge nel momento preciso in cui egli prende coscienza della perdita del privilegio piú importante di cui godesse: il "furore" divino, l'ispirazione poetica, il "sacer furor," tanto celebrato nei Nutricia. ${ }^{21} \mathrm{La}$ reazione immediata di Orfeo è infatti quella di scagliarsi innanzitutto contro il "furore," e, solo in un secondo tempo, anche contro il fato e la morte: ". . . O mio furore, / o ciel nimico, o Morte!" (Vv. 313-14). La presa di coscienza della perdita dell'essenza vitale del 'canto,' il furore poetico, lo condurrà inevitabilmente alla morte, la quale si spiega in rapporto diretto alla poesia, che ne determina il sacrificio.

L'inconsolabile dolore di Orfeo, dunque, non è dovuto alla perdita definitiva di Euridice, bensí alla consapevolezza di aver perso il dono della poesia, di non poter piú riuscire a comporre versi, di non poter piú intessere un "canto" che possa esprimere il dolore della sua perdita: "Qual sarà mai sí miserabil canto, / che pareggi el dolor del mio gran danno?" (Vv. 322-23). Non esiste nessuna indicazione nel testo che ci impedisca di attribuire il "gran danno" alla perdita del dono della poesia anziché di Euridice. Se ci si vuole attenere esclusivamente al testo concreto della Fabula invece che alla conoscenza tradizionale del mito di Orfeo, su cui si sono sempre basate le indagini degli studiosi, ci si accorge che, dopotutto, non c'è menzione alcuna, né nel testo dei versi né nelle frasi esplicative degli avvenimenti, interposte in vari punti, ${ }^{22}$ del fatto che Orfeo si sia realmente girato a guardare Euridice. A prescin- 
dere dall'espressione, messa in bocca a Mercurio, nei versi che annunciano la favola - "Orfeo . . . tra via drieto si volse" (vv. 9, 11) - versi che non fanno parte integrante del testo della narrazione o dell'azione, il poeta non fa nessuna allusione all'atto del voltarsi di Orfeo. Questo particolare, sfuggito alla critica, può solo essere interpretato intenzionale da parte del Poliziano, il quale se ne serve per dare maggior risalto al valore e al ruolo della poesia nell'Orfeo. Omettendo questo particolare, il poeta fa sí che, nel testo, avvenga il passaggio diretto ed immediato dal canto trionfale della poesia sulla morte alla seconda morte di Euridice, che viene strappata ad Orfeo da una Furia.

Lo strappo di Euridice appare essere la vendetta immediata degli dei, contro il poeta, per il suo atto di proclamazione della vittoria della poesia. Tenendo presente che le Furie (Erinni, nella mitologia greca) erano, fin dall'età omerica, spiriti di vendetta, intesi a proteggere gli ordini sociale, naturale e cosmico, e che una delle loro funzioni era quella di vendicarsi contro i vati che svelavano troppo apertamente i segreti al profano, il fatto che sia stata una Furia ad arrestare irrevocabilmente il tentativo di Orfeo di voler recarsi nuovamente all'inferno potrebbe indicare che Orfeovate, ${ }^{23}$ Orfeo-poeta sia stato vendicato per aver voluto svelare, con il suo canto trionfale, la vittoria della poesia sulla forza delle divinità infernali; la Furia, per vendetta dell'atto imperdonabile, gli toglie proprio il soggetto e l'oggetto del suo trionfo: la poesia.

Il poeta tracio, il fondatore della poesia e della civiltà, non può accettare di diventare un uomo come gli altri: la perdita delle differenze, come nella tragedia greca e nella religione primitiva, crea ostacoli all'armonia e genera una reazione violenta. ${ }^{24}$ La perdita della voce di Apollo rappresenta una minaccia non solo per il poeta, ma per l'ordine culturale stesso: venendo a mancare la poesia, i valori della società si dissolvono, il disordine e il caos ritornano a regnare, e gli esseri ricadono nella loro bassa condizione, librandosi agli istinti ed alla volgarità, come dimostra l'atroce strazio compiuto dalle Baccanti. Solo alla poesia, simbolo piú alto della civilizzazione è concesso il potere di illuminare le anime, liberandole dalla bassa condizione, di dominare il mondo e di creare l'armonia universale: tale è il significato del miracolo del canto di Orfeo al cospetto di Plutone e degli altri esseri infernali. Solo la poesia può godere di valore atemporale, sopravvivere alla morte, e innalzarsi sul caos dell'universo - come il canto trionfale recitato all'uscita dall'inferno e il canto dell'ode saffica latina, simbolo della poesia piú dotta ed erudita, e riservata all'élite. 
La presenza dell'importanza dello strazio delle Baccanti, nella favola, è stata sempre sottovalutata. Per alcuni, il tono dell'intero passo sull'aspetto fisico della morte ed il fatto che Orfeo sia ucciso da Baccanti ebbre servirebbero ad accentuare "the completely irrational element involved in death. ${ }^{25}$ Moltissimi altri relegano il valore del passo ad un interesse puramente stilistico-linguistico, mettendone in rilievo la rapidità del "rythme endiablé," "la violence des rimes en -ezza," "l'excitation rythmique de tout le passage, morcelé par les répétitions," e lo ritengono "le morceau le plus brillant et le plus original de la pièce," quello in cui la tecnica del Poliziano "fait merveille."26

Se è la forza della violenza che sembra rivelarsi nell'atto dello sparagmòs (stracciamento), l'essenziale rimane dissimulato, cioè la scelta libera della vittima e il sacrificio che restituisce l'unità e l'armonia. Quando Orfeo non ha piú il dono della poesia, decide di affermare la sua volontà in un atto che potrebbe chiamarsi esistenziale: sceglie di andare contro la vita e la sfida abbracciando la morte. Il tentativo di ricatto degli dei si risolve nella decisione di estraniarsi dal mondo comune dei mortali, di cui si rifiuta di accettarne il destino, e si concretizza nel rifugio in un mondo che, pur macchiato dalla turpitudine di un amore innaturale, è pertanto conforme al mondo degli dei, al mondo di Giove e di Apollo:

Fanne di questo Giove intera fede, che dal dolce amoroso nodo avinto si gode in cielo il suo bel Ganimede, e Febo in terra si godea Iacinto. (Vv. 346-49)

Trasformandosi in un nuovo Narciso, scegliendo l'omosessualità, va logicamente incontro a tutte le consequenze che una tale decisione comporta: negazione della procreazione e dunque della vita stessa, accettazione della cristallizzazione dell'essere nell'identico e nell'affezione di se stesso. Ma la sua scelta non è altro che il rifiuto volontario dell'alternativa di una vita caotica o altrimenti pietrificata, di una vita senza poesia, ossia di una vita già non piú vita; è dunque il rifiuto della morte stessa. Come ha scritto Lorenzo de' Medici, nel suo noto commento all'Orfeo del Poliziano (partendo, però, da una premessa opposta alla nostra): "E però il principio della vita è la morte della vita non vera." $27 \mathrm{Ed}$ Orfeo sceglie la vita vera, cioè la poesia, scelta che comporta la morte della vita non vera. Ma la scelta della morte è la negazione stessa della morte. L'atto di affermazione della volontà di Orfeo è 
l'atto supremo di tentativo di liberazione dalla vita senza poesia e della proclamazione del valore atemporale dell'ultima, il cui trionfo si realizza a pieno nel libero e volontario atto del poeta, che vuol dire scelta della poesia alla morte. Il sacrificio di Orfeo diventa cosí la proclamazione estrema della vittoria della poesia sulla morte. "Pel Caos onde tutto el mondo nacque" (v. 280) aveva proclamato nella preghiera a Plutone - in seno al caos si compie il sacrificio di Orfeo, e sul caos risorgerà come nei Nutricia, l' 'eloquenza trionfatrice' ("facundia victrix," v. 120) e si effonderanno 'le parole e il suono" ("vocemque verba," v. 29) del Vate.

Un Orfeo che, ispirato, deve il suo trionfo iniziale al potere della poesia, un Orfeo che, abbandonato dal "furor" divino, affida ancora e sacrifica tutto se stesso alla poesia, pare realizzare a pieno il mito simbolico del poeta tracio, non solo, ma anche aderire fedelmente al neoplatonismo ficiniano. Per queste ragioni, mi sembra poco convincente la lezione del Branca, secondo la quale Orfeo non avrebbe "nessuna di quelle responsabilità simboliche, platoniche, di senso metafisico, di cui lo caricava Ficino, ${ }^{28}$ teoria da cui non si scosta molto la presa di posizione degli altri studiosi, tra cui la Maïer, secondo la quale "On ne saurait charger cet Orphée, très humble, somme toute, de responsabilités symboliques ... rien ne permet d'affirmer que l'auteur ait songé à exprimer dans la pièce l'édifiante léçon prônée par Ficin."29

Che la Fabula sia tutta tesa all'esaltazione della poesia e che il sacrificio di Orfeo deva spiegarsi in rapporto alla poesia risulta evidente anche dall'urto dei mondi, creati dal poeta, in cui si muovono i vari personaggi. L'Orfeo si apre con una visione del mondo pastorale: il sereno, incontaminato pacifico mondo di un'Arcadia stilizzata, forse il mondo dell'età dell'oro, la cui armonia non è turbata né dalla narrazione dello smarrimento del 'vitellin' di Tirsi (vv. 17-19; 93-100), né dalla descrizione dell'insana passione di Aristeo (vv. 29-31; 44-48), che, anzi, si distendono nella musica dei versi. È il mondo dove regna il mito dell' 'eterna primavera,' ritratto nella spensieratezza dei pastori, nella giovinezza e nella "belleza snella" di Euridice, che di "neve e rose ha 'l volto e d'or la testa" (vv. 71, 107-08). Un mondo già compiuto e perfetto in sé non ha bisogno di articolarsi drammaticamente, onde le linee semplici dell'esordio. Ma un mondo pieno d'equilibrio, privo di caos, è un mondo ideale, irreale, da favola; e la realtà ideale può solo essere frutto di un'invenzione umana, immaginaria, un'utopia, o una creazione poetica estetica, una visione, un'illusione insomma che, irreale che sia, personifica pertanto una perenne 
aspirazione dell'uomo, un qualcosa che non può mai essere distrutto completamente e che egli non può mai ripudiare interamente, come il giardino ariosteo di Alcina - che sembra già fissato nei versi della Fabula - la concezione del quale, nella fine analisi di Bartlett-Giamatti, potrebbe descrivere anche, nella sua dicotomia di illusione e realtà che pervade tutto il Cinquecento, il mondo arcadico e ideale creato dal Poliziano:

deceptive though its illusions were, Alcina's garden remains as the image of a way of life which man can never wholly reject . . . because it is so much part of himself: . . . it embodied a way of life which should be avoided, it also embodied something which can never be destroyed; and, therefore, no other garden, virtuous or divine . . can ever replace it. ${ }^{30}$

Un tale mondo è necessariamente costretto a rivelare la sua precarietà, a dileguarsi, a contatto con il mondo reale, che proromperà nei suoi aspetti piú concretamente umani: la morte di Euridice (momento che, dal punto di vista strutturale, coincide con l'inizio della seconda metà dell'opera: dal v. 198 in poi), e in quelli piú privilegiati: la poesia ed il sacrificio del poeta. Con il mondo pastorale, si dileguano e spariscono completamente dalla narrazione anche tutti i suoi personaggi, di cui il poeta lo aveva gremito. Le considerazioni di ordine metafisico, che perseguono il poeta tracio, non riguardano né Mopso, né Tirsi, né l'altro "amatore," Aristeo, il quale, con l'entrata in scena di Orfeo è tagliato completamente fuori dalla narrazione. Questo particolare della sua misteriosa sparizione, generalmente sorvolato o visto come segno di incoerenza formale e di contenuto, è, invece, rigorosamente consequenziario della logica polizianesca. Aristeo fa parte del mondo idillico, ideale, che, per definizione, esclude e l'alta 'poesia e la tragedia. Egli si dilegua nel momento preciso in cui il suo mondo irreale viene ad urtarsi con il mondo privilegiato della poesia, la cui visione si apre con l'apparzione di Orfeo sulla sommità del monte, recitando la saffica latina, simbolo di un'alta espressione di un ideale di cultura e di civilizzazione. Alla visione del pacifico mondo pastorale della prima parte della Fabula, si opporrà la visione caotica della conclusione che, con la fragorosa irruzione delle Baccanti, impegnate a realizzare il verdetto degli dei, segna il momento culminante in cui il mondo di Orfeo viene ad urtarsi con il mondo sovrumano che esse rappresentano.

La presenza del mitico mondo pastorale, all'inizio della favola, si spiega non tanto per la contribuzione alla rievocazione delle vi- 
cende tradizionali del mito di Orfeo, quanto per il maggior significato che contribuisce a conferire al ruolo della poesia. Se, nel finale orgiastico delle Baccanti, che rappresentano l'umanità librata alla volgarità ed agli istinti, il Poliziano ritrae l'aspetto caotico del mondo senza poesia, nel mondo pastorale, ne ritrae quello cristallizzato: benché ideale, in quanto esclude la violenza e riflette il mito dell'eterna primavera, è pietrificato poiché esclude anch'esso la poesia e la cultura. ${ }^{31}$

La dicotomia dei mondi della Fabula - da una parte l'irreale, pastorale e il caotico, dionisiaco, entrambi scevri di poesia, e dall'altra il reale di Orfeo, dominato dalla poesia - si riflette nella struttura simmetrica dell'opera che ne afferma la continuità contingente e che tutta converge nel sacrificio di Orfeo. I motivi, i temi, le immagini, le strutture stilistiche della prima parte sono puntualmente ripresi, sviluppati, ed intensificati nella seconda, dove trovano quasi sempre o un contrapposto, o una spiegazione, o la conclusione estrema. Se esigenze di spazio non mi permettono di enunciarli ed analizzarli nel presente saggio, ${ }^{32}$ si rilevi almeno, anche se in un modo non troppo esauriente, che il senso della morte e del sacrificio è già implicito nelle immagini e nelle descrizioni iniziali. L'episodio del "vitellin" smarrito di Tirsi, "bianco / che ha una macchia nera in sulla fronte / e duo pié rossi e un ginocchio e 'l fianco" (vv. 17-19), che è unanimemente considerato solo una giustapposizione o uno spunto divertente, inserito per conferire "sensualità coloristica" ${ }^{33}$ o "local color," ${ }^{34}$ ha un significato profondo.

Le espressioni contenute nella narrazione di Tirsi, dopo aver ritrovato il vitellino, "collo mozo," "sbudellato," "dar di cozo," "pieno el gozo" (vv. 94-96, 98), annunciano rispettivamente la testa mozza di Orfeo, lo strazio compiuto sul suo corpo dalle Baccanti che erano precisamente "imbottate come pevere" (v. 374), mentre il "mugghiar là drieto al monte" degli armenti (p. 22) annuncia il fragoroso rumore delle Baccanti. Al "silenzio" della prima parola del primo verso della Fabula, si contrappongono le urla sfrenate degli ultimi versi, "Bacco, Bacco, eú, oé. . . ." A prescindere da queste corrispondenze, ed a prescindere anche dal simbolismo dei colori del vitellino, premonitori degli avvenimenti che seguiranno, ${ }^{35}$ è la figura stessa del vitello, simbolo della vittima degli antichi sacrifici pagani, che prefigura il sacrificio di Orfeo. Nella narrazione, il vitellino errabondo non è toccato dalla sciagura ed è "raviato" da Tirsi "nella mandria" a cui appartiene (v. 96); come Aristeo, come Mopso ed il suo padrone, si ritira an- 
ch'esso nel mondo irreale. La sua immagine, però, ritorna a vivere nello strazio finale, dove i suoi colori realizzano il loro significato simbolico e profetico: il nero la morte, il rosso il sacrificio, il bianco la rinnovazione. Nel mondo pastorale, ideale, l'immolazione non avviene - la morte non esiste in un mondo che non ha poesia ma ha luogo nel mondo concreto, dove si muore quando viene a mancare la poesia. Al vitello, antica vittima dell'immolazione pagana, si sostituisce l'uomo, simbolo del sacrificio cristiano.

Forse non sarebbe troppo ardito vedere Orfeo come il Cristo della poesia, che si sottomette silenziosamente e senza ribellione alcuna allo scempio compiuto dalle Baccanti, dall'umanità librata agli istinti, priva della luce della poesia, per poterla redendere dalla sua bassa condizione, ridandole la poesia, la creazione poetica, l'unico tipo di attività umana suscettibile di fare regnare l'ordine sul caos della terra. Contrariamente all'Orfeo dei Nutricia, che supplica le donne trace, sue carnefici, con dolce canto e con pietosi accenti - "Frustra suave melos frustra pia verba moventem" (v. 299) - l'Orfeo della Fabula muore senza nessun attimo di esitazione e senza emettere nessun lamento.

Se la rassegnazione della sua morte appare, come ha alluso Apollonio, in un certo senso cristiana, ${ }^{36}$ il modo in cui si esplica il sacrificio è prettamente pagano, e il fatto che sono delle Baccanti ad eseguire l'uccisione di Orfeo ci riconduce alla loro divinità, Dionisio, il cui corpo fu lacerato dai Titani. Come Dionisio che, secondo i neoplatonici, rinasce interamente ed è l'anima dell'universo che, benché divisa, ritiene la sua indistruttibilità, ${ }^{37}$ cosí Orfeo ritiene indistruttibile l'anima del mondo della poesia, la quale risorgerà, si rinnoverà e si perpetuerà tramite il suo sacrificio. Come la fenice che, con la morte, rinasce piú bella di prima. ${ }^{38}$

Dopo il Carducci che, continuando a definire l'Orfeo una "rappresentazione," rilevò che il suo contenuto, "per l'argomento patetico e per la catastrofe" era già simile in misura maggiore o minore a quello della tragedia, ${ }^{39}$ voci solo sporadiche hanno accennato al carattere tragico della Fabula, senza pertanto elaborare le loro asserzioni. Apollonio, per esempio, scrive che "movendo da un lieto principio, e concludendosi con un amaro fine, la favola si rivela tragedia in regola con le vecchie definizioni e con le forme che stanno affacciandosi." 40 Orlando, sostenendo che il "momento piú alto" dell'Orfeo consiste nella "straordinaria, singolare imitatio compendiaria della tragedia greca," nega alla favola valore drammatico: "Idillio o dramma dunque? Né l'uno né l'altro, ma 
un'opera affrettata, incompleta, e pur tanto geniale"; però riconosce "una truce scena di tragedia" nello strazio di Orfeo, la cui sorte gli ricorda quella toccata a Penteo nelle Baccanti di Euripide. ${ }^{41}$ All'infuori di queste limitate allusioni, gli studiosi non vedono né tragedia né dramma nella Fabula, come la Maïer, la quale pensa che Orfeo non vada oltre "l'image et la fonction du 'troubadour' dont la voix peut charmer toutes les oreilles. . . . La passion et le drame sont complètement absents. Voilà, en bref, pour le négatif." 42

È sconcertante che l'Orfeo venga escluso completamente dal mondo della tragedia. Se si sostiene, con Girard, che la tragedia è l'equivalente dei veri riti e che ha dunque un carattere di sacrificio - "un caractère sacrificiel" - e che possiede necessariamente "une face maléfique, dionysiaque dira Nietzche, liée à sa création, et une face ordonnatrice bénéfique, apollinienne, dès qu'on entre dans la mouvence culturelle," ${ }^{43}$ allora $1^{\prime}$ Orfeo risulta chiaramente una tragedia. È la tragedia del sacrificio del poeta vate, il quale si oppone all'aspetto 'malefico, dionisiaco,' caotico, e si sacrifica per ripristinare quello 'ordinatore benefico, apollineo.' La Fabula, oltre a contenere entrambi questi aspetti, mette in azione proprio i due antipodi: da un lato Apollo, nella veste di Orfeo, dall'altro Dionisio, nel ruolo delle Baccanti. Nella fragorosa irruzione dei canti orgiastici, nell'urto del mondo di Orfeo con il mondo sovrumano, rappresentato dalle divinità bacchiche, culmina e si traduce a pieno l'antitesi Apollo-Dionisio. L'uccisione di Orfeo, che si svolge conformemente ai riti dionisiaci, assume l'aspetto di un rito di sacrificio: lo sparagmòs (stracciamento), è eseguito con la partecipazione unanime delle Baccanti all'immolazione, e lo smembramento della vittima è effettuato per mano delle carnefici senza l'utilizzazione di nessun'arma.

Essendo quasi di rigore, quando si parla del binomio ApolloDionisio, nel campo della tragedia, riferirsi alle opinioni contrarie di Platone e di Aristotele, è opportuno inquadrare l'Orfeo nella cornice platonica o aristotelica. Tenendo presente la nota definizione aristotelica della tragedia e delle sue virtú catartiche, e l'opposizione di Platone al disordine tragico ed alla violenza tragica che, secondo lui, non potrebbero assurgere a sinonimo di ordine e di armonia, si nota che la Fabula non esclude né l'una né l'altra e che tende, anzi, a conciliare ambedue le posizioni. La visione neoplatonica del ruolo civilizzatore e dominatore della poesia, rifulgente nei versi latini della saffica, nel canto di Orfeo che piega gli spiriti infernali, e nei versi della celebrazione del trionfo della 
poesia sulla morte, è seguita dal drammatico atto, di tipo aristotelico, catartico e purificatore dell'uccisione di Orfeo.

In un certo qual modo aristotelica si presenta la vittima stessa: come l'eroe tragico di Aristotele, che deve possedere, per essere degno di definirsi tale, oltre alle virtú, anche un 'difetto tragico,' una debolezza qualunque che possa rendere inoperante la sua virtú e permettere cosí allo spettatore di librarlo all'orrore e alla morte, cosí Orfeo, il vate che ha incantato e civilizzato il mondo con la virtú della sua parola, ha ceduto ad una comprensibile e perdonabile debolezza umana, prima girandosi a guardare Euridice contro il monito di Plutone (atto che, anche se come ho detto sopra non è inserito nel testo, è pertanto tradizionalmente sottinteso dallo spettatore, dal lettore) - debolezza che non diminuisce lo spirito della pietas, entro cui deve svolgersi la tragedia - e ad un'imperdonabile e meno comprensibile debolezza dopo (incomprensibile per lo spettatore, s'intende), scegliendo di seguire l'omosessualità, una vita che va contro le leggi fissate dalla natura stessa dell'uomo. Potrebbe essere quest'ultimo il 'difetto tragico' che permette allo spettatore di librarlo alla vendetta delle Baccanti.

Similmente all'uccisione di Penteo, che si presenta "à la fois comme le paroxysme et la liquidation d'une crise provoquée par le dieu lui-même, comme une 'vengeance' suscitée par l'incroyance des Thébains et surtout de sa propre famille," uccisione che serve a ristabilire l'ordine e la pace in Tebe, ${ }^{44}$ la morte di Orfeo, il quale è provocato anche lui, meno direttamente, dal Dio infernale, con il rilascio condizionato di Euridice:

Io te la rendo, ma con queste legge

ch'ella ti siegua per la cieca via, ma che tu mai la sua faccia non vegge

fin che tra' vivi pervenuta sia (vv. 294-97)

e l'ammonimento: "dunque 'l tuo gran desire, Orfeo, corregge" (v. 298) - che esprime la consapevolezza, da parte di Plutone, dell'inevitabile debolezza a cui cederà Orfeo - appare simultaneamente come il frutto di un'azione divina e la consequenza di una libera decisione della vittima. Se il dramma, nella violenza atroce dello sparagmòs e nell'apparente effetto catartico, sembra aderire alla posizione aristotelica, quell'atto violento e purificatore è inteso a ristabilire, al tempo stesso, l'ordine culturale nel mondo, il risorgere della poesia e l'affermazione del suo trionfo sul caos, ossia a restaurare il mondo neoplatonico della seconda 
parte della Fabula dominato da Orfeo. Nel suo attuarsi, è la tragedia aristotelica stessa che porta al conseguimento dei valori perseguiti dalla posizione platonica. In altre parole, il platonismo consegue l'affermazione ed il trionfo dei suoi valori, servendosi di un mezzo - la violenza tragica - che esclude per definizione. Il Poliziano parte da Platone per ritornare a Platone, tramite Aristotele.

Se non si può troppo facilmente sostenere con il Branca che l'Orfeo sia "una fabula nel senso aristotelico e filologico e momariesco ... non in quello simbolico del platonismo ficiniano," non bisogna sottovalutare l'importanza che sia lo studioso che il Pirrotta attribuiscono al senso teatrale dell'opera, per cui lo spettacolo verrebbe a coinvolgere lo spettatore in modo tale da farlo diventare "quasi attore," ${ }^{\prime 6}$ trasformazione che presuppone, ovviamente, l'identificarsi e l'immedesimarsi nei sentimenti e nelle vicende rappresentati sul palcoscenico. Non è difficile capire come lo spettatore, cattivato dalla rappresentazione scenica, anziché essere immerso nella lettura del testo, sia portato a scorgere nell'opera, per l'effetto della pietas che lo coinvolge, soprattutto la dimensione umana che essa ritrae: la morte prematura di una bellissima donna e la disperazione fino alla follia del consorte.

Mentre sul piano ideologico ed artistico, la morte di Orfeo diventa la scelta ed il sacrificio del poeta per l'esaltazione e la rinnovazione della poesia, sul piano umano, atto a commuovere e toccare direttamente lo spettatore, rappresenta l'atto estremo di ribellione dell'essere umano contro l'assurdità del destino, e che si concretizza nella libera scelta di un amore innaturale, ossia di una vita contraria alle leggi fissate dalla natura e dagli uomini stessi, di cui deve accettarne le consequenze. Cosí, anche sul piano umano, la morte diventa in un certo senso sacrificio, in quanto è il risultato inevitabile della scelta consapevole e volontaria dell'amante.

Dal momento in cui gli viene annunciata la morte di Euridice, si assiste allo sgretolamento graduale dell'equilibrio psicologico e mentale di Orfeo. Si può osservare come venga meno e ceda il posto, poco a poco, al parossismo, prodotto dalla tensione sempre piú soffocante, provocata dal groviglio interno delle passioni e che lo condurrà alla disfatta definitiva. Affiorato, in un primo tempo, nella reazione immediata alla notizia della morte dell'amata, espressasi sotto la forma di mesta rassegnazione nell'elegiaco ed umanissimo compianto ("Dunque piangiamo, o sconsolata lira / . . . / piangiam mentre che 'l ciel ne' poli aggira," vv. 198-200), seguito dall'affermazione della sua incertezza circa la propria capa- 
cità di controllare il dolore ("come potrò soffrir mai dolor tanto?" v. 203), a cui subentra, prima, una momentanea e vaga intenzione di morire ("sanza te non convien che 'n vita stia," v. 205) e, poi, la subitanea decisione di recarsi alle "tartaree porte" (v. 206), per "provar se la giú merzé s'impetra," (v. 207), la tensione è ora acuita dalla certezza della propria impossibilità di resistere al dolore ("né posso piú resistere al dolore," v. 257) e dalla decisione di morire ("Dunque rendete a me la mia speranza," v. 276, e "se pur me la nieghi iniqua sorte, / i' non vo' su tornar ma chieggio morte," vv. 284-85) - dove le espressioni "né posso piú," "dunque," "chieggio," rappresentano la risoluzione rispettiva delle indecise "come," "non convien," "se."

Dopo la perdita definitiva di Euridice, il parossismo progredisce con un ritmo sempre piú incalzante ed intensificato del pathos. Orfeo non si lamenta tanto del fatto che gli sia stata tolta la donna amata, quanto di non poter trovare consolazione al suo dolore, di non potere, quindi, trovare l'equilibrio dentro di sé ("Qual sarà mai sí miserabil canto .. .'" vv. 322-25). All'angoscia della consapevolezza dell'inconsolabilità del suo "mortale affanno," si aggiunge quella della certezza della futilità di qualsiasi tentativo che voglia mettere in atto. Sentimenti che, dischiusi dalle dolci ed accorate parole di Euridice ("Ohimé che 'l troppo amore / n'ha disfatti ambedua," vv. 306-07, e "ben tendo a te le braccia, ma non vale," v. 310) e confermati dalle profetiche espressioni della Furia, che arresta l'ultimo tentativo di Orfeo di riconquistare l'amata ("e di te stesso omai teco ti dole; / vane sono tue parole, / vano el pianto e '1 dolor: tua legge è ferma," vv. 319-21), esplodono nell'affermazione della presa di coscienza del proprio furore (". . . O mio furore," v. 313) e nell'atto di rivolta contro gli dei, che impongono il loro verdetto inscrutabile agli uomini, senza un motivo umanamente comprensibile, e contro l'umanità.

È qui che la sua tragedia assurge a simbolo universale, poiché diventa la tragedia di tutta l'umanità. Ad infrangere la legge sovrumana non è Orfeo-poeta, è Orfeo-uomo che, in quanto tale, dotato di tutte le debolezze dei mortali, cede, da essere umano e quindi imperfetto, ad un attimo d'impulso. Orfeo-poeta poteva ristabilire l'ordine sulla terra e dominare la volontà degli dei; Orfeo-uomo non può controllare nemmeno il caos della propria passione e raggiungere l'equilibrio psicologico e mentale. Orfeouomo è, come il resto dei mortali, succube alle leggi imposte ai mortali. Anche il suo amore soccombe alla morte dal momento 
che gli amanti stessi non possono sfuggire e trascendere la temporalità imposta su di loro dalla morte.

La presa di coscienza di Orfeo-uomo dell'esistenza del suo "furore" umano, la follia, coincide con la presa di coscienza di Orfeopoeta della perdita del suo "furore" divino, l'ispirazione poetica. Se Orfeo-uomo offre allo spettatore una tragica visione della condizione umana, che si risolve in un atto di ribellione contro la sovrumanità e l'umanità stessa, Orfeo-poeta offre al lettore la sua tragedia piú intima, la tragedia del poeta, che si concretizza nella scelta di un atto gratuito, il sacrificio, inteso ad effettuare l'ideale di ricreazione secondo l'humanitas.

Solamente il poeta può, con la virtú della sua parola, illuminare e civilizzare gli esseri - come farà nei Nutricia - non solo, ma può, con il suo sacrificio, dominare e ordinare il mondo caotico degli istinti, della volgarità, della violenza (personificato dalle Baccanti) e ricreare l'armonia universale. Cosí la Fabula diventa l'inno alla poesia, la cui vittoria è personificata nel canto del vate e nella sua scelta dell'atto estremo: scelta della poesia alla morte, ossia negazione della vita senza poesia.

$\mathrm{Nel}$ suo tentativo di conciliazione di mondi opposti e di tendenze diverse - la conciliazione della fede negli ideali classici con le sollecitazioni cristiane, la fatalità della tragedia greca con la facoltà del libero arbitrio, il platonismo con l'aristotelismo - il Poliziano racchiude suggestivamente nell'Orfeo l'ideologia dell'Umanesimo in un suo complesso momento critico.

Piú delle altre opere del Poliziano, l'Orfeo deve considerarsi il piú autentico messaggio della sua filosofia e della sua poetica, la piú viva testimonianza della sua umanità, e la piú fervida professione di fede nella virtú fondatrice, ricreatrice, e atemporale della poesia: "Inlibata . . . / Carmina numquam ullis parcarum obnoxia pensis." 47

\section{The University of Georgia}

\section{NOTE}

1 Per un'approfondita illustrazione della poetica della docta varietas del Poliziano, ved. E. Bigi, La cultura del Poliziano e altri studi umanistici. Saggi di varia umanità, 5 (Pisa: Nistri-Lischi, 1967), pp. 90-101.

2 Q.v. "Quo argumento dicendū Vergilius, non Virgilius," Cap. LXXVII, di Angeli Politiani Miscellaneorum Centuria Prima, e la seconda e la terza epistola del liber V dell'Epistolarum - "Barptolomaeus Scala Ang. Politiano suo S. D." e "Angelus Politianus Barptolemaneo suo S. D." - in Angeli Politiani Opera Omnia, Basilea 1533 (rist. dell'ed. di Venezia: Aldo Manuzio, 1498), rist. in An- 
gelus Politianus, Opera Omnia, a cura di I. Maïer (Torino: Bottega d'Erasmo, 1971), tomo I, rispettivamente a pp. 286-87, 60, e 62.

3 Per l'ispirazione dell'Orfeo dalle momarie veneziane, ved. V. Branca, Poliziano e l'umanesimo della parola. Saggi, 655 (Torino: Einaudi 1983), pp. 55-72. Per una dettagliata illustrazione delle fonti della Fabula di Orfeo, ved. E. Bigi, "Umanità e letterarietà nell' 'Orfeo' del Poliziano," Giornale storico della letteratura italiana, 159 (1982), 183-215. Si vedano anche il commento di N. Sapegno al testo della Fabula di Orfeo, nell'ed. da lui curata (A. Poliziano, Rime. Testo e nota di N. Sapegno, $2^{a}$ ed. riveduta e corretta [Roma: Edizioni dell'Ateneo, 1967]), pp. 107-30; il commento di G. Carducci al testo della Favola di Orfeo, nella sua ed. (Le Stanze, l'Orfeo e le rime di Messer A. Ambrogini Poliziano, rivedute . . . e illustrate, 2a ed. [Bologna: Zanichelli, 1912]), pp. 371-91; il commento di G. Trombadori al testo di La Favola di Orfeo, nella sua ed. (A. Poliziano, Le Stanze, l'Orfeo e le rime. Passi scelti, con introd., commento e appendice di pagine critiche [Milano: Vallardi, 1933]), pp. 65-79. Ved. anche I. Maïer, Ange Politien. La Formation d'un poète humaniste (1469-1480), Travaux d'humanisme et Renaissance, LXXXI (Ginevra: Droz, 1966), pp. 405-15, e S. Orlando, "Note sulla 'Fabula di Orfeo' di Angelo Poliziano," Giornale storico della letteratura italiana, 143 (1966), 503-17.

4 E. Bigi, "Umanità e letterarietà nell"'Orfeo' del Poliziano," cit., 204.

5 L. Russo, "L'Orfeo del Poliziano," Belfagor, 8 (1953), 280-81.

6 Il Russo stesso riconduce poi l'Orfeo ad un'ispirazione "melodiosamente idillica" (cit., 270). Cosí N. Sapegno, che scopre nell'opera "un idillio, appena sottolineato da un senso di vaga e quasi inconsapevole mestizia" ("Il sentimento umanistico e la poesia del Poliziano," Nuova Antologia, 17 [1938], rist. in Pagine di storia letteraria [Palermo, 1966], p. 226) e M. Santoro, che vi scorge un idillio, percorso da "una malinconia pensosa e soave," la quale sembrerebbe costituire "il motivo piú autentico dell'umanità dei personaggi" (Poliziano e l'umanesimo. Lezioni dell'anno accademico 1960-61 [Napoli: Libreria Liguori, 1961], p. 243). Non molto diversa è l'interpretazione di G. Trombadori, che nega carattere umano alla "favola": "I sentimenti non vibrano nell'Orfeo con corda umana, ma rivivono in eterno puri, trasferiti nell'ideale regione del mito. . . . Orfeo, come tutti i personaggi della Favola, non è stato sentito e reso nella sua verità umana" ("Poliziano," 1933, rist. in Saggi critici [Firenze, 1950], p. 111). E. Rho va oltre e, sottolineando l'aspetto fiabesco dell'opera, la colloca in "un'atmosfera di irrealtà fanciullesca" (La lirica di Agnolo Poliziano [Torino-Genova: Lattes \& C., 1923], p. 102), mentre, per D. De Robertis, essa è frutto di "pura immaginazione, quale era stata consegnata alla tradizione da Virgilio e da Ovidio, pura 'favola' cioè appunto spettacolo, diletto," che "non esce dai termini di una festa mantovana" (La formazione poetica del Poliziano e "Le Stanze per la Giostra." Corso tenuto dal Prof. D. De Robertis. Anno Accademico 1962-1963, [Cagliari: Libreria Universitaria Cagliaritana, 1963], p. 76). N. Pirrotta, mettendo felicemente in evidenza il "gusto letterario, figurativo, e musicale" dell'autore, ravvisa nel personaggio di Orfeo "il simbolo o la personificazione di una piú alta concezione della poesia come canto" ( $\mathrm{Li}$ due Orfei. Da Poliziano a Monteverdi. Con un saggio critico sulla scenografia di E. Povoledo, $2^{a}$ ed. [Torino: Einaudi, 1975], pp. 15, $22,23)$.

7 E. Bigi, "Umanità e letterarietà nell' Orfeo del Poliziano," cit., 193, 192, 191.

8 "Chi amasse i procedimenti armonici difficilmente saprebbe resistere alla tentazione di collocare sotto il segno d'Orfeo la linea ideale di sviluppo del Poliziano, facendo convergere il tema del dramma volgare e i Nutricia, de poetica et poetis, la Sylva che raccoglie una specie di professione di fede nella virtú formatrice della poesia" (E. Garin, "L'ambiente del Poliziano," in Istituto Nazionale di Studi sul Rinascimento, Il Poliziano e il suo tempo. Atti del IV Convegno internazionale di studi sul Rinascimento [Firenze: Sansoni, 1957], p. 37).

9 lbid. 
10 Q.v. vv. 181-88 di "Angelus Politianus: Ad Fontium," in Bartholomaeus Fontius (B. Della Fonte), Carmina, ed. J. Fógel e L. Juhász (Lipsia: Teubner, 1932), p. 27 (rist. in Opera Omnia, ed. I. Maier, cit., tomo 3, p. 172).

11 "Finis erat dapibus: citharam pius excitat Orpheus, / Et movet ad doctas verba canora manus. / Conticuere viri, tenuere silentia venti; / Vosque retro cursum mox tenuistis, aquae: / Jam fessis pendere sub aethera pinnis, / Jamque truces videas ora tenere feras ....," Manto, vv. 13-27; "An vero ille ferox, ille implacatus et audax / Viribus, ille gravi prosternens cuncta lacerto, / Trux vitae, praeceps animae, submitteret aequo / Colla jugo aut duris pareret sponte lupatis / $\mathrm{Ni}$ prium indocilem sensum facundia victrix / Vimque reluctantem irarum flatusque rebelles ...." Nutricia, vv. 116-38; ". . Nec fabula mendax / Parrhasio lapides movisse Amphiona plectro, / Orpheos atque lyram curva de valle secutas / In caput isse retro liquido pede fluminis undas; / Cumque suis spelaea feris, cum rupibus ipsis / Dulcia pierias properasse ad carmina fagos . ..." $\mathrm{Nu}$ tricia, vv. 283-320, in A. Poliziano, Le Selve e la Strega. Prolusioni nello Studio fiorentino (1482-1492), ed. I. Del Lungo (Firenze: Sansoni, 1925). Citazioni successive dal testo dei Nutricia sono tratte da questa edizione.

12 Ved. M. Ficino, Opera (Basilea, 1576): I, p. 670, Epist. lib. I, Alexandro Braccio; p. 927, Epist. lib. IX, Petro Divitio; p. 1323, In convivium.

13 Per esempio, Epist. lib. I, Antonio Canisiano, in Opera, cit., I, p. 651.

14 Ved. Opera, I, p. 318, Theol. Platon., XIV, 8.

15 Che la preoccupazione della morte sia uno degli elementi essenziali del mondo poetico del Poliziano è stato indicato da piú di uno studioso. J. Cotton-Hill, per es., afferma che "death is the central theme of his truest and most spontaneous poetry, written on occasions of death, such as the Elegy for Albiera (1473), interrupted by death, such as the Stanze per la Giostra (1478), or a legend (favola) of death such as the Orfeo (1480)" tanto da diventare quasi un'ossessione. L'autrice, però, non estende la sua investigazione al campo letterario e si sofferma piuttosto sulle possibili cause che determinarono la morte concreta del poeta ("Death and Politian," Durham University Journal, 46, No. 3, (1954), 96-97). E. Donato sostiene che la morte sia la 'forza dominante' dell'universo poetico del Poliziano e, pur alludendo, all'inizio del saggio, alla presenza nell'opera, del rapporto tra la morte e la poesia, il ruolo dell'ultima non viene discusso nel suo commento, che diventa, in effetti, una coronazione della forza del destino $\mathrm{e}$ della futilità di qualsiasi sforzo umano per controllarlo, e si conclude con la proclamazione della staticità dell'azione e dell'irrazionalità della morte, che sarebbero inerenti all'opera ("Death and History of Poliziano's Stanze," Modern Language Notes, 80 [1965], 27-40).

16 ". . . Cosí desideravo ancora io che la fabula di Orfeo . . . fusse di subito, non altrimenti che esso Orfeo, lacerata, cognoscendo questa mia figliuola essere di qualità da far piú tosto al suo padre vergogna che onore, e piú tosto atta a dargli malinconia che allegreza ...," nella lettera di "Angelo Poliziano a

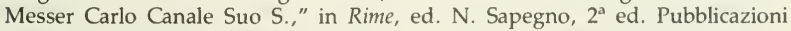
della scuola di filologia moderna dell'Università di Roma, 12 (Roma: Edizioni dell'Ateneo, 1967), p. 107.

17 Cito da A. Poliziano, Rime, ed. N. Sapegno, cit. Da quest'edizione sono tratte anche tutte le citazioni successive del testo della Fabula di Orfeo.

$18 \mathrm{~V}$. Branca, per es., nel suo recente volume, nella saffica non scorge che il senso teatrale, e la considera una di "quelle prepotenti e elegantissime inserzioni di cortigianerie" (Poliziano e l'umanesimo della parola, cit., p. 63).

19 L. Russo, cit., p. 278. Ved. simile descrizione dell'effetto del canto di Orfeo in Nutricia, vv. 116-31; in Elegia al Fonzio, vv. 181-88, in B. Della Fonte, Carmina, ed. cit., n. 19, p. 172.

20 A. Poliziano, "Latini dettati a Piero De' Medici," in Prose volgari inedite. Poesie latine e greche edite e inedite di A. Ambrogini Poliziano, raccolte e illustrate da I. Del Lungo (Firenze: G. Barbera, 1867), pp. 38, 40. 
21 Ved. Nutricia, vv. 139, e 146 e sgg.

22 Si vedano soprattutto quelle che seguono, rispettivamente, i vv. 137, 301, 305, 317.

23 Orfeo è esplicitamente chiamato 'vate' altrove dal Poliziano, per es., nei $\mathrm{Nu}$ tricia: "Indulsit vati Eurydicem ...." (v. 296).

24 Ved. R. Girard, La Violence et le sacré (Paris: Grasset, 1972): "Dans la religion primitive et la tragédie un même principe est à l'oeuvre. . . . L'ordre, la paix et la fécondité reposent sur les différences culturelles. Ce ne sont pas les différences mais leur perte qui entraînent ... la lutte. . . . Comme dans la tragédie grecque donc, comme dans la religion primitive, ce n'est pas la différence, mais bien sa perte qui cause la confusion violente" (pp. 77-79).

25 E. Donato, cit., p. 36.

26 I. Maïer, Ange Politien. La Formation d'un poète humaniste (1469-1480), cit., pp. 409 e 411 . S. Orlando nota che la "rima difficile - "disprezza - spezza - scavezza; morte - forte; fora — mora . . ." - ricorda quella dantesca dei Malebolge (cit., p. 514). F. Tateo, sostenendo che la "tragica morte di Orfeo, punito dalle Baccanti . . . suggerisce il pezzo piú originale di tutta la favola, quello del concitato canto delle Baccanti, che riprende, con un nuovo esperimento formale, il gusto per la descrizione delle scene bacchiche nelle Stanze e ritroveremo nel Rusticus," trova che "la presenza di questo canto" sia "sostanzialmente in contrasto con la conclusione tragica della vicenda" e ritiene opportuno doverla giustificare, spiegandola "con l'occasione del carnevale, quello del 1480, in cui forse la 'favola' fu composta"! (Lorenzo de' Medici e Angelo Poliziano. Letteratura italiana Laterza, 14 [Bari: Laterza, 1972], p. 135). Anche G. Trombadori ritiene che "v'è solo concitazione ritmica e una certa violenza delle rime in -ezza. . . . Ma tragedia non v'è" (cit., p. 111).

27 Lorenzo de' Medici, Opere (Bari: Laterza, 1913), p. 25. Lorenzo rimprovera ad Orfeo l'incapacità di non essere veramente 'morto' e di non aver cosí potuto raggiungere la perfezione della felicità: "Ed arebbe Orfeo tratto Euridice dell'inferno e condottola tra quegli che vivono, se non fussi rivoltosi verso l'inferno: che si può interpretare Orfeo non essere veramente morto, e per questo non essere aggiunto alla perfezione della felicità sua, di avere la sua cara Euridice. E però il principio della vera vita è la morte della vita non vera. . . ."

28 V. Branca, cit., p. 63.

29 1. Maïer, cit., p. 395. Ved. anche, per es., S. Orlando, il quale sostiene che il mito di Orfeo "non assume valore di simbolo in questa favola o rappresentazione scenica. Occorre bandire ogni interpretazione allegorica" (cit., $516 \mathrm{n}$.).

30 A. Bartlett-Giamatti, The Earthly Paradise and the Renaissance Epic (Princeton: Princeton University Press, 1966), p. 164.

31 A questo proposito, non può non ritornare alla mente il passo di Dante, in cui, prendendo spunto da alcuni versi di Ovidio (Metam. XI, 1-2), pone ad esempio il mito di Orfeo per esporre ciò che intende per senso allegorico; dopo aver richiamato il talento del "savio uomo," che "con lo strumento de la sua voce," faceva "mansuescere e umiliare li crudeli cuori," conclude che "coloro che non hanno vita di scienza e d'arte . . . sono quasi come pietre" (Convivio, II, I, 3).

32 Ho elaborato altrove quest'aspetto dell'opera, in un saggio dove ho affrontato, per la prima volta, l'argomento svolto nel presente saggio e ne ho sviluppati altri, quali la questione del libero arbitrio, della fortuna, e della virtus nell'Orfeo. Ved. M. Cocco, "Poesia e morte nell'Orfeo del Poliziano," in Origini del dramma pastorale in Europa. Centro di studi sul teatro medioevale e rinascimentale. Atti dell'VIII Convegno di Studi. Viterbo, 31 maggio - 3 giugno 1984 (pubblicazione imminente: maggio 1985).

33 E. Cecchi, "L'Orfeo," in La critica letteraria, ed. M. Acrosso (Palermo: Palumbo, 1970), p. 177. 
34 E. Donato scrive che "Mopso's lost calf adds a light realistic tone, so often present in Poliziano's shorter poems. . . . Mopso and his calf . . . are here only for local color" (cit., 34, 37).

35 Senza avviare un troppo ovvio discorso sul simbolismo dei colori, si potrebbe almeno suggerire che il rosso dei "piedi" annunci la morte di Euridice, morsa al piede da un serpente ("da un serpente venenoso e reo, / ch'era fra l'erbe e' fior, nel pié fu punta," vv. 194-95), il rosso sul "ginocchio e 'l fianco" il sangue del corpo di Orfeo, lacerato dalle Baccanti. La "macchia nera in sulla fronte" potrebbe rappresentare il presentimento funesto della doppia morte di Euridice e di quella di Orfeo.

36 M. Apollonio sostiene che sia "la tranquillità di chi è in possesso dell'eterno" ("Paesaggio dell'Orfeo," in Istituto nazionale di studi sul Rinascimento, Poliziano e il suo tempo, cit., p. 29).

37 Per un approfondimento dell'argomento, rinvio a I.M. Linfirth, The Arts of Orpheus (1941) (New York: Arno Press, 1973), pp. 318 sgg.

38 È da segnalare l'Orfeo, fenix de Turia, opera spagnola di autore anonimo, la quale sviluppa appunto il tema della fenice nel mito di Orfeo (Ms. della Biblioteca Nacional di Madrid, $14.600 / 30^{\circ}$, stampato in Appendice a P. Cabañas, El mito de Orfeo en la letteratura española [Madrid: Consejo Superior de investigaciones cientificas, 1948]), pp. 333-83.

39 G. Carducci, "Delle poesie toscane di Messer Angelo Poliziano. Discorso," in A. Ambrogini Poliziano, Le Stanze, l'Orfeo e le rime, cit., pp. $90 \mathrm{sgg}$.

40 M. Apollonio, cit., p. 76.

41 S. Orlando, pp. 513, 515.

42 1. Maïer, cit., p. 413, Per E. Donato, "La Favola di Orfeo is not a tragedy, it is not even drama" (cit., 36). Anche secondo M. Santoro, "manca la tragedia e manca il dramma" (cit., p. 243). G. Trombadori sostiene che "La tragedia non c'è perché Orfeo ... non è stato sentito e reso nella sua verità umana" (cit., p. 111).

43 R. Girard, cit., p. 405.

44 Ibid., pp. 185-86.

45 V. Branca, cit., p. 64.

46 Ibid., p. 63. Ved. N. Pirrotta, cit., pp. 14-15.

47 'Vergin carme non soggetto mai a fuso alcun di Parca' (Nutricia, vv. 30-31). 